\title{
LabonaChip
}

\section{D Arrays of Microcages by Two-photon Lithography for Spatial Organization of Living Cells}

\begin{tabular}{|r|l|}
\hline Journal: & Lab on a Chip \\
\hline Manuscript ID & LC-ART-11-2018-001240.R2 \\
\hline Article Type: & Paper \\
\hline Autho Submitted by the & 23-Jan-2019 \\
\hline Complete List of Authors: & $\begin{array}{l}\text { Larramendy, Florian; Albert-Ludwigs-Universitat Freiburg Institut fur } \\
\text { Mikrosystemtechnik } \\
\text { Yoshida, Shotaro; University of Tokyo, Institute of Industrial Science } \\
\text { Maier, Daniela; Albert-Ludwigs-Universitat Freiburg Institut fur } \\
\text { Mikrosystemtechnik } \\
\text { Fekete, Zoltan; Albert-Ludwigs-Universitat Freiburg Institut fur } \\
\text { Mikrosystemtechnik, Microsystems Materials Group } \\
\text { Takeuchi, Shoji; The University of TOKYO, CIRMM/IIS } \\
\text { Paul, Oliver; Albert-Ludwigs-Universitat Freiburg Institut fur } \\
\text { Mikrosystemtechnik }\end{array}$ \\
\hline
\end{tabular}




\title{
3D Arrays of Microcages by Two-photon Lithography for Spatial Organization of Living Cells
}

Florian Larramendy ${ }^{\mathrm{a}}$, Shotaro Yoshida ${ }^{\mathrm{b}}$, Daniela Maier ${ }^{\mathrm{a}}$, Zoltan Fekete ${ }^{\mathrm{a}}$, Shoji Takeuchib,c and Oliver Paul ${ }^{\mathrm{a}}$

${ }^{a}$ Department of Microsystems Engineering (IMTEK), University of Freiburg, Germany

${ }^{\mathrm{b}}$ Center for International Research on Integrative Biomedical Systems, Institute of Industrial Science (CIBiS-IIS), The University of Tokyo, Japan

${ }^{\mathrm{c}}$ International Research Center for Neurointelligence (WPI-IRCN), The University of Tokyo Institutes for Advanced Study (UTIAS), The University of Tokyo, Tokyo, Japan.

\begin{abstract}
This paper addresses a nanoengineering approach to create fully three-dimensional (3D) network of living cells, providing an advanced solution to in vitro studies on either neuronal networks or artificial organs. The concept of our work relies on stackable scaffolds composed of microcontainers designed and dimensioned to favor the geometrically constrained growth of cells. The container geometry allows cells to communicate in the culture medium and freely grow their projections to form a 3D arrangement of living cells. Scaffolds are fabricated using two-photon polymerization of IP-L 780 photoresist and coated with collagen. They are stacked by mechanical micromanipulation. Technical details of proposed nanofabrication scheme and assembly of the modular culturing environment is explained in details. Preliminary in vitro results using PC12 cells have shown that these structure provides a good basis to healthy cell growth for at least 16 days. Our approach is envisioned to provide
\end{abstract}


tailor-made solutions of future 3D cell assemblies for potential applications in drug screening or creating artificial organs.

Keywords: Neuronal cell cages, Two-photon polymerization, Direct laser writing, Threedimensional cell network, PC12 cells, Stackable scaffolds. 


\section{Introduction}

In vitro models of the human brain have started to deploy their potential in the development of assays for neurological disorders [1] such as Alzheimer's [2] and Parkinson's diseases [3] or Amyotrophic lateral sclerosis [4]. The ability of microstructured devices to help mimic the organization of the neural tissue at the single cell level opens up new routes to physiologically relevant in vitro models $[5,6]$.

$2 \mathrm{D}$ cultures are commonly used, due to its cost effectiveness, easy handling, and robustness across diverse cell types $[7,8,9,10]$. There were attempts relying on the integration pillars and walls as secondary microstructures to go beyond the limitations of 2D models $[11,12,13,14]$. Microchannels with rough hydrogel sidewalls were introduced into culturing chambers by Kunze et al. [15]. Microfluidic flow-chip holding concave microwells were proposed by several groups [16,17]. 2D cell cultures with microwells entrapping air bubbles for patterning the microenvironment were demonstrated by Goral and coworkers [18]. Microwell-meshes for mimicking 3D environment were tested for anti-drug screening [19].

In spite of its popularity, these $2 \mathrm{D}$ cultures are considered inadequate in recapitulating specific physiological features due to the limitations in cell-cell and cell-extracellular matrix interactions [20, 21]. In contrast, $3 \mathrm{D}$ cultures provide a more complex artificial environment with longer lifespan, also being more informative and predictive, therefore they are considered better complement of animal models. The work of Takahashi et al indicated more extensive connectivity can be seen in the neuronal evolution using 3D constructs, which results in a more synchronous electrical activity [22]. Bosi et al reported the ability of 3D geometry to improve functional organization and synchronization in small neuronal assemblies [23]. A group utilizing graphene scaffolds gave experimental evidence that 3D neuronal networks are very different from 2D networks [24]. Using Polystyrene fiber scaffolds, Kim et al demonstrated that 3D environment defined by fiber arrays significantly influenced neuronal cell morphology and outgrowth pattern [25]. In PDMS microlattice scaffold, Li and co-workers found a higher neuron-to-astrocyte ratio and a higher degree of cell ramification in 
the $3 \mathrm{D}$ culture compared to the $2 \mathrm{D}$ culture due to the change of scaffold topography [26]. Improved tissue organization of 3D cultures can be also exploited in the promising field of organ-on-a-chip platforms [27] or drug discovery [28].

The past decade has witnessed the development of new technologies potentially enabling 3D neuronal models representative of reality. Two families of methods with this aim can be identified. They rely on either soft or hard biomaterials for controlled cell culture. In the soft biomaterial methods, cells are not rigidly supported. These methods include the aggregation of cells [29,30] and cell culture in hydrogel $[12,31]$ and gellan gum [32]. Soft substrates are advantageous in that cells are free to grow in any direction and are exposed to little mechanical stress. Furthermore, the cell culture environment approximates the softness of biological tissues. However, these techniques produce random 3D neuronal cultures and cells can sediment on the supporting substrate if the gel is too soft [12]. In contrast, an excessive gel viscosity hinders cells growth and the development of connections [32].

In the techniques using hard biomaterials, in contrast, cells are usually supported by solid scaffolds. This includes cell cultures on silk [33], graphene [34], PDMS [35], and photoresist scaffolds [36], silicon and PDMS microwells [37,38], and glass microbeads [39]. Several reasons speak in favor of rigid 3D scaffolds: they are better approximations of the natural extracellular environment [40,41], they enable higher structural complexity [33], and they offer advantageous physical properties [40] and increased cell survival [42]. In addition, 3D scaffolds allow to control the attachment and proliferation of cells as well as the number of cells per sample [43]. For these reasons, the present study relies on solid scaffolds designed to favor the development of 3D neuronal networks.

Two-photon polymerization (2PP) of photosensitive materials [44, 45] has been demonstrated as an attractive technique for creating controlled miniaturized 3D scaffolds and has already been used for cellular studies [14, 36, 46-48]. However, it has served mostly for creating small fixed 3D objects 
on substrates for $2 \mathrm{D}$ or $2.5 \mathrm{D}$ cellular studies. In this paper, we propose to use $2 \mathrm{PP}$ to create transferrable and stackable 3D architectures for fully 3D cellular arrangements and cellular culturing.

The basic concept was sketched in a previous conference publication [49]. In the present study, we report in significantly greater detail on stackable photoresist scaffolds made by 2PP and tailored for the development of controlled 3D cellular networks. The geometric principles underlying the structures and the technologies used to fabricate them are described in details. Preliminary in vitro experiments are presented to show biocompatibility and neurite growing through the cage structure.

\section{Microscaffolds made of cell cages}

\subsection{Concept}

Our novel method is based on the regular arrangement of cell cages shaped as shown in Figure 1a. The basic geometric unit is a truncated octahedron consisting of hexagonal and square elements with identical edge lengths. The shape is the same as the Wigner-Seitz cell of a body-centered cubic lattice in solid-state physics [50]. Several reasons led to the selection of this particular geometry: (i) It is close to spherical, its facets are smaller than its cross-section, and it is thus expected to be well-suited as a cell cage; (ii) it allows gapless packing in 3D; (iii) a cut through its center defines roughly hemispherical container structures (Figure 1b) which can be periodically extended into structural layers to be finally stacked into 3D scaffolds. Moreover and in contrast with previously reported structures [10], the scaffolds can be designed to have a small contact area with the substrate, which helps an easy detachment of the whole structure from the substrate.

The concept of confined cell growth is demonstrated in this study with PC-12 cells. We will not distinguish between axons and dendrites, and subsequently term all PC-12 cell projections as neurites. After fabrication of the structural layers, cells are deposited on them and allowed to settle and grow in their hemispherical containers. Cell-populated structural layers are then stacked one on top of the other. This is repeated several times to obtain a 3D scaffold populated by cells in regularly arranged 
containers. Thereafter, neurites are free to grow and establish connections between neighboring cells. Considering the container geometry, neurites projecting from a cell soma can chose from among fourteen openings in order to establish such connections. Cell bodies are expected to stay in their fixed position. This way, the cell container has an influence on the evolution of cells. The entire process is described in Section 5 Experimental.

\section{2. $3 \mathrm{D}$ microstructure}

Geometrical models were developed using the computer-aided design tool SolidWorks. As suggested by Figure 1c, thickening the edges makes the structures more robust. At the same time, the vertical columns of the original structure cut into half pieces are equipped with connection pins on one side and corresponding connection holes on the other side. Similar half-columns are added on top of the upper structure and on bottom of the lower one, in order to enable multi-layer stacking. The hemispherical unit structure of Figure 1c is extended periodically in 2D, as shown in Figure 1d. Although the two structural layers are basically mirror images of each other with respect to a horizontal plane, it is useful to make the distinction between them, in view of their different pin/hole arrangements. We term them structures of types A and B (see Figure 1c). In multi-layer stacks, structures of the two types are stacked in alternation.

Structures with cell cage diameters of $30 \mu \mathrm{m}, 40 \mu \mathrm{m}, 50 \mu \mathrm{m}$ and $60 \mu \mathrm{m}$ were realized in order to identify dimensions suitable for healthy cell development. In the case of the $50-\mu \mathrm{m}$ cages, the bars forming the edges of the truncated octahedron have a diameter of $10 \mu \mathrm{m}$. For the other cell cage diameters, the edge bar dimensions were scaled linearly.

Each cell cage is composed of six square frames and eight wider, hexagonal openings. In order to hinder cells from escaping through these wider openings, additional barrier lines crossing them were implemented (Figure 1d). This approach effectively blocks the migration of cell soma, while leaves sufficient space for cell-to-cell communication between neighboring cages. For the 50 - $\mu \mathrm{m}$-diameter 
cages, the barrier bars have a diameter of $5 \mu \mathrm{m}$, while those of the other cage diameters were scaled, accordingly.

In addition, alignment blocks consisting of a large cuboid $\left(50 \times 50 \times 38 \mu \mathrm{m}^{3}\right)$ with protruding and recessed alignment crosses on opposite sides, shown in Figures 1e and 1f, serve for mechanically manipulation and alignment of the structures with respect to each other. Such alignment blocks were added, either on a single side of the structure or in pairs on opposite sides. The used 2PP direct laser writing (DLW) system has a writing area of up to $100 \times 100 \mathrm{~mm}^{2}$, which in principle provides enough freedom to scale up the fabrication process to create a number of cages in a single fabrication batch. The concept of 2PP is illustrated in Figure S1 in the Supplementary Information. However, when one relies on the high-speed 3D printing option, which was the case in this work, the area is reduced to $200 \times 200 \mu \mathrm{m}^{2}$. For this reason, the basic $2 \times 2$ unit of Figure $1 \mathrm{~d}$ was applied. Larger structures were realized by stitching these units. Stitching errors of the fabrication process were estimated and found to be less than $1 \mu \mathrm{m}$ in all cases. The structures in Figures $1 \mathrm{e}$ and $1 \mathrm{f}$ consist of four and eight such units, respectively.

\section{Experimental}

\subsection{Microstructure fabrication}

Microstructures were designed using SolidWorks and preprocessed using DeScribe and NanoWrite, two Nanoscribe GmbH in-house developed softwares, into a format suitable for the 2PP DLW system (Photonic Professional by Nanoscribe GmbH). The system is equipped with a femtosecond pulse $780 \mathrm{~nm}$ laser focused into a droplet of IP-L 780 photoresist (Nanoscribe $\mathrm{GmbH}$ ), as shown in Figure 2a. This resist contains pentaerythritol triacrylate (>95\%) and 7-(diethylamino)-3-(2thienylcarbonyl)-2H-1-benzopyran-2-one $(<5 \%)$. The exposure is performed through a glass substrate (Nanoscribe $\mathrm{GmbH}$ ) by means of an oil-immersion objective with a numerical aperture of 1.4. Photoresist polymerization properties were investigated at laser powers ranging from $4 \mathrm{~mW}$ to 
$16 \mathrm{~mW}$. Type A and B structures were fabricated with solely their connection pins in contact with the glass substrate, as shown in Figure 2b.

Thanks to this minimum substrate contact, the structures can be easily detached from the substrate. The structures were polymerized using a laser power of $11 \mathrm{~mW}$. The scan speed was $30 \mu \mathrm{m} / \mathrm{sec}$, resulting in an in-plane width of exposed lines of about $0.3 \mu \mathrm{m}$ and an out-of-plane thickness of about $1 \mu \mathrm{m}$. For the alignment blocks a power of $13 \mathrm{~mW}$ was used, producing slightly wider lines. On each substrate, 16 structures were fabricated at a time, alternating structures of type A and B, separating them by a gap of $500 \mu \mathrm{m}$. The glass substrate was then immersed for 20 min in PGMEA at RT and rinsed with IPA. After drying, the samples were inspected by optical microscopy (Olympus IX, Olympus) with regard to their shape and number. Finally, SE microscopy was performed on each sample. Only cell cages of perfect shape were transferred to in vitro experiments.

\subsection{Preparation for cell culture}


The biocompatibility of the IP-L resist has been demonstrated previously [38, 48-49]. We nevertheless took particular care to minimize any possible cytotoxicity of the scaffold material, following an established four-step protocol [51-52] to prepare the substrate and scaffold structures for cell culture. The first step is to remove residual photoresist using IPA. Secondly, any previously unexposed photoresist trapped within the structures is thoroughly polymerized by subjecting the structures to a one-minute UV flood-exposure at $365 \mathrm{~nm}$ wavelength. The third step is then a thermal anneal at $150^{\circ} \mathrm{C}$ for 30 min eliminating solvent still trapped in the photoresist. Finally, an $\mathrm{O}_{2}$ plasma treatment renders the photoresist surface and glass substrate hydrophilic. This last step is performed just before attaching a 3-mm-high silicone rubber frame on the glass substrate, surrounding the 16 structures, as shown in Figure 2c. The frame defines a culture chamber with a surface area of $1 \mathrm{~cm}^{2}$. The height of the silicone rubber is compatible with the insertion angle of the micromanipulator used to grab and stack the microstructures.

\subsection{PC12 cell culture}

With the purpose of favoring the controlled attachment and growth of cells, cell-adhesionpromoting collagen in phosphate-buffered saline (PBS) solution (ten times diluted Dulbecco's PBS $10 \times$, D1408, Sigma-Aldrich) is next dispensed into the silicone chamber (Figure 2c). After rinsing, the structures are densely covered by collagen, as schematically shown in Figure $2 \mathrm{~d}$. All these steps are realized with great caution in order to avoid the unwanted detachment of the structures from the substrate. The cell culture experiments were performed using PC12 cells (RCB0009, Riken Bio Resource Center) that had undergone fewer than ten passages with Dulbecco's Modified Eagle's Medium (DMEM) (D5796, Sigma) supplemented with 1\% (v/v) penicillin-streptomycin (P4333, Sigma), 10\% (v/v) fetal bovine serum (Biosera), and 10\% (v/v) horse serum (Japan Bio Serum). Neuron-like PC12 cells were seeded onto the collagen-coated structures with DMEM supplemented with $1 \%$ penicillin-streptomycin and $10 \mathrm{ng} / \mathrm{ml}$ nerve growth factor (N6009, Sigma). About 
30,000 cells $/ \mathrm{cm}^{2}$ were dispensed. Again, the cell deposition is realized cautiously to avoid the detachment of structures. Incubation was carried out for 3 hours under standard conditions for neuronlike PC12 cells, i.e. $5 \% \mathrm{CO}_{2}$ at $37{ }^{\circ} \mathrm{C}$, allowing cells to settle in the containers, as illustrated by Figure 8e. After this duration, type A and B structures are sequentially stacked on top of each other (Figure 8f). Incubation was then continued for one more day before inspection. After optical microscopy, neuron-like PC12 cells were visualized with the LIVE/DEAD® Viability/Cytotoxicity Kit for mammalian cells (L3224, Molecular Probes). In this test, viable cells are labeled with calcein AM detectable as green fluorescence, whereas dead cells are labeled with ethidium homodimer (EthD-1) responding by red fluorescence. Excitation of the live stain occurs at $494 \mathrm{~nm}$ with fluorescent emission at $530 \mathrm{~nm}$. For the dead stain, the excitation is at $528 \mathrm{~nm}$, with fluorescent emission at $617 \mathrm{~nm}$.

\subsection{Scaffold stacking}

In order to stack cell-carrying structures, we had to combine the micromanipulator equipment with a microscope (IX81, Olympus, Japan) compatible with the culture chamber. The experimental equipment used for stacking the cell-carrying layers is shown in Figure S8 of the Supplementary Information. The culture chamber (stage-top incubator INUBH-ONICS-F1, Tokai Hit, Japan) is controlled to have a $\mathrm{CO}_{2}$ concentration of $5 \%$. A Peltier element keeps temprature at $37{ }^{\circ} \mathrm{C}$. Substrates with the silicone-bounded culture chamber are extracted with their microscaffolds and cell cultures from the incubator and inserted into the culture chamber 30 min before the stacking process starts. This latency time reduces the stress on the cells caused by transportation. Then the substrate is aligned with the axis of the micromanipulator so that the alignment blocks of the microstructures can be reliably grabbed using the micromanipulator. The culture chamber is then opened and the micromanipulator is inserted into the cell culture. First, all type B structures are stacked onto type A structures in cell culture medium to avoid having to flip the structures over and to keep cells alive. 
Thereafter, stacks with three and four layers are built analogously. It was occasionally observed that cells adhered to alignment blocks and the micromanipulator, making it difficult to release the structures from the micromanipulator. In such cases, we opened the micromanipulator slowly while avoiding misaligning the structures; we then gradually lowered the manipulator before retracting it, using the lower alignment block as a fixed support for detaching it from the upper structure. This manipulation took 15 minutes and, despite all caution, caused occasionally misalignment of structures. To give a precise interpretation of our results, misaligned assemblies were omitted from the in vitro evaluation.

\subsection{Cell fixation for scanning electron microscopy}

For fixation, the culture medium was removed, after which the chips were rinsed with PBS solution and incubated in 4\% glutaraldehyde solution (Sigma-Aldrich) for 12 hours at $4{ }^{\circ} \mathrm{C}$. Then, the glutaraldehyde solution was removed and the chips were again rinsed with PBS solution. For cell dehydration, we used a cascade of aqueous ethanol solutions with increasing ethanol concentrations. The cells were thereby incubated in 50\%, 70\%, 80\%, 90\% and 100\% ethanol for 10 minutes each. After the last dehydration, the fixed samples were air-dried at RT for a day to remove alcohol residues before inspection using an SE microscope (SU-8000 FESEM, Hitachi High-Technologies Co., Japan). Amazingly healthy-looking cells are the result, without apparent degradation or collapse of their delicate structure that might be expected from a drying procedure without critical-point drying. This preparation method for SE microscopy was already successfully applied in studies of nanoscale topography on neurite development of PC12 cells [10]. Further technical details are reported in [53].

\section{Results}




\subsection{Structure optimization and stacking}

The concept and optimization of the scaffold structures represented a significant part of this study. The three stages of the structural evolution are illustrated in the Supplementary Information, in particular Figure S2, form a delicate ancestor via a robust barrier-free intermediate structure to the final structure equipped with cell-migration hindering barriers.

The larger structures of Figures $1 \mathrm{e}$ and $1 \mathrm{f}$ were realized by stitching the basic $2 \times 2$ unit structure with an appropriate overlap, further details of which are provided in Figure $\mathbf{S 3}$ of the Supplementary Information. As a result of such stitching, parts of the structure were exposed up to three times, without apparent loss of structural resolution. Variants of the final optimized stacking layers are shown in Figures 3a and $3 b$ and in greater detail in Figure $\mathbf{S 4}$ in the Supplementary Information.

Due to their small dimensions, stacking the structures is a challenge. Larger structures (with widths $>500 \mu \mathrm{m})$ would be easier to handle. However, writing them using the $2 \mathrm{PP}$ instrument $[26,37-38]$ necessitates unrealistically long writing times. For this reason, we initially limited this work to samples as shown in Figures $3 \mathrm{a}$ and $3 \mathrm{c}$ formed of 16-container structures. The writing time of these structures was still $45 \mathrm{~min} / \mathrm{sample}$. The structures were handled using a micromanipulator [54] comprising microtweezers operated by electrostatic actuation (AOI Electronics. Co., Ltd., Japan) and whose $x, y$, and $z$ motion was monitored under a microscope. The actuation of the microtweezers was controlled with micrometer resolution, with a maximum gap width of $100 \mu \mathrm{m}$. In the absence of cells and in order to avoid flipping the structures over and subjecting them to uncontrolled movements due to static electricity, the stacking was carried out in isopropyl alcohol (IPA). Water was found to be less suitable because of its stronger capillarity. Once a structure has been grabbed by its alignment block, it is positioned and aligned above another. The upper block is then lowered onto that of the lower structure. Once the structures are in close contact, the upper structure is released. A storyboard of the stacking steps is provided by Figure S5 in the Supplementary Information. 
Observing stacked structures by scanning electron (SE) microscopy turned out to be challenging. Indeed, after stacking, type A and B structures are mechanically locked thanks to the alignment blocks and connection holes and pins. However, their connection is not strong enough to keep them reliably in place during transportation from the micromanipulator setup to the SE microscope. Accelerations, vibrations and air flow contributed to the frequent disassembly of the stacked structures. As a countermeasure, we kept stacked structures in IPA and let them dry at room temperature (RT), after fixing the glass substrate on the SE microscope holder. Stacking results with formed cages are shown in Figures 3c-e.

During structure optimization, mechanical stress turned out to be a challenge. Initial structures were consistently bent, as illustrated by Figure 4a. Such bending can be evidence of a stress gradient. A first hypothesis was therefore that the stress resulted from the geometry of the structures in combination with the particularities of the Nanoscribe technology, where the laser beam passes through already cross-linked volumes. The recommended solution in such cases is to adapt the writing speed and exposure power. However, even though we observed a slight improvement, such optimization could not entirely fix the problem. As a next possible cause, we hypothesized the IPL 780 photoresist used in this study to be the cause of the stress. This photoresist behaves similarly to the well-established SU-8 photoresist [55]. 2PP process steps are known to stress SU-8 photoresist [56-57] and pre- and post-exposure bakes have been suggested to alleviate the problem [57-58]. Consequently, we performed such thermal tests, from which we concluded that a pre-bake is not a helpful option because it reduces the achievable structural resolution. Likewise, a post-bake is no option either, since the stress was observed to remain unchanged. As a third possibility, we considered the well-known fact that SU-8 is stressed by its exposure to certain solvents [59]. Consequently, we conjectured that the stress in the IP-L 780 structures originated in their development. Initially we used IPA as the developer to remove unexposed photoresist, based on its mention in previous studies [6061], and therefore suspected this solvent to be responsible for the observable stress. After we switched 
to the alternative developer propylene glycol methyl ether acetate (PGMEA, MicroChem), before rinsing with IPA, we obtained structures without apparent stress, as illustrated by Figure 4b.

\subsection{D Culture}

In preparation of the 3D cell culture, two preliminary studies were performed. The first aimed at quantifying the distribution of cells per cell cage as a function of the cage diameter, while the second was to evaluate the efficiency of the cell barriers.

Ideal for controlled cell culture would be to have exactly one cell grow in each cage, while offering them enough room to prosper. In order to assess how well this vision can be realized, samples with the different cage diameters were prepared. On each substrate, four structures of each cage diameter and 32 cages per sample were produced and the same cell culture (with approximately 30 000 cells $/ \mathrm{cm}^{2}$ ) was dispensed on the entire set. The cell culture protocol is reported in Section 5 Experimental. After five days of culture, we performed the LIVE/DEAD ${ }^{\circledR}$ test. Results were collected on 6 samples per cage diameter and are summarized in Figures 5a-d. for the four diameters from $30 \mu \mathrm{m}$ to $60 \mu \mathrm{m}$. Figure $4 \mathrm{e}$ gives the occupancy distribution per container. In the case of the 30 $\mu \mathrm{m}$ containers, it is rarely observed that two or more cells occupy a container, but in view of Figure 5a, even single cells seem to be somewhat squeezed in their narrow environment. In the case of the 60- $\mu \mathrm{m}$ containers, at the other end of the tested diameter range, cells have enough space but are often more than two per container. In-between, $40-\mu \mathrm{m}$ and $50-\mu \mathrm{m}$ containers seem to best fulfill the condition of hosting one cell with the highest probability. For the rest of the study, among these two sizes we chose to use the 50 - $\mu \mathrm{m}$-diameter cages leaving cells with sufficient room for healthy development.

For the second study, we designed structures with $50-\mu \mathrm{m}$-diameter cages, with and without cell barriers. The development of cell cultures on these two variants was then compared. Cells in solution are rather small, with bodies around $10 \mu \mathrm{m}$ in diameter. For this reason, cells escaped from the barrier- 
free cell cages, taking advantage of their freedom of motion. In contrast, cells cultured for two days on structures equipped with barriers have adhered to the containers. We thus concluded that barriers effectively hinder the passage of cells from one cage to the next. Nevertheless, openings are large enough ( 3 to 5 - $\mu$ m-wide) to let cells develop neurites freely in all direction. Time-lapse movies in Figure S6 of the Supplementary Information show that cells are free to move within their container but do not leave it. Cells move over the grids formed by the cell barriers but do not cross them.

The biocompatibility of the structures was then tested. To this end, cell cultures were exposed to three conditions for up to 16 days: (i) cell culture on a standard cell culture dish as a reference, (ii) cell culture on a collagen-coated flat glass substrate, and (iii) cell culture on scaffold structures equipped with barrier lines on a glass substrate, all coated with collagen. Results of conditions (ii) and (iii) are shown in Figure 6. Cells were observed to be alive after 16 days. Moreover, in both cases they developed neurites. A LIVE/DEAD ${ }^{\circledR}$ test was applied thereafter. The test revealed that no more cells are dead on and around the scaffold structures than on the flat glass substrate. Even better, a large number of cells has obviously migrated onto the structures, showing their strong affinity for the polymeric structures (Figure 6).

\subsection{Stacked structures and cell culture in $3 \mathrm{D}$}

The next experiments aimed at going into the third dimension. For this purpose, we again relied on structures with $50-\mu \mathrm{m}$-diameter cages with barriers. Each substrate comprised 16 scaffolds with 32 cages in a checkerboard arrangement, alternating eight structures of each type A and B separated from each other by $500 \mu \mathrm{m}$. The structures were treated and packaged with homemade silicone rubber chamber, cells were cultured, and scaffolds were stacked according to the protocol described in Section 5. Stacking tests were carried out with durations of 5, 8, 10, 12, and 15 min per stacking step. The tests revealed that the yield in live cells after stacking and incubation decreases if the stacking process exceeds $12 \mathrm{~min}$. On the other hand, longer stacking times allow better control of the stacking 
process. As a trade-off, we therefore chose a stacking time of 10 min. After stacking, the culture chamber was closed again and cells were let in culture for an extra hour. During this period, using time-lapse optical microscopy, we monitored that cells did not escape from the scaffolds. Thereafter the substrates were returned to the incubator for one more day. After completion of the stacking protocol, the tip of the micromanipulator was cleaned ultrasonically in $70 \%$ ethanol.

A LIVE/DEAD® test was then applied to verify the viability of the cells. The confocal optical micrographs of Figure 7 show there is a large number of live cells on and near a triple stack of structures (Figure 7a) whereas only one dead cell is found on the structure (Figure 7b). Figures 7a and $7 \mathrm{c}$ show that polymeric structures are fluorescent. The combination of the information of Figures $7 \mathrm{a}-\mathrm{c}$ on Figure $7 \mathrm{~d}$ is an attempt to enhance the contrast between cells and scaffold.

SE microscopy offers the most detailed view of the structures, as illustrated by Figure 8. Stacked structures with three and four layers of cell culture are shown in Figures 8a and 8b, respectively. Potential cell-to-cell connections by neurites between layers and between cages are visible in Figure 8c. As a consequence, 3D neuronal networks are expected to form on these multi-level structures. Even with the largest structures (32 containers and two alignment blocks), we did not succeed to go beyond four layers, because the microtweezers are not high enough to grab more than two superposed layers together. Moreover, it turned out to be too difficult to align and settle additional layers on stacks of four layers without dismantling them.

On the other hand, we found it unproblematic to transfer stacked structures populated by cell cultures into the SE microscope. Indeed, by their adhesion to the structures, live cells exert strong cohesive forces between structural layers, keeping them well attached to each other. Moreover, the network of neurite interconnections between scaffold layers constitute solid biological links effective against the disconnection of the stacks. Remarkably, these cohesive effects remained active throughout the protocol for preparing the cell cultures for SE microscopy. Further evidence of cell adhesion is provided in Figure $\mathbf{S 8}$ of the Supplementary Information, where cells cause the adhesion of a cell culture on a single-layer scaffold to the silicone sidewall of a culture chamber. 


\section{Conclusion}

In this paper, we demonstrated a new approach to enable artificial 3D cell networks. The concept is based on stacking layers of cell-sized, container-shaped microcages designed for hosting cell cultures. We optimized the hemispherical microcages to host most likely a single cell and to keep it in place, while allowing it to extend neurites to cells in neighboring cages. The structures comprise features allowing them to be stacked layer by layer, thus forming an arrangement of microcages with the periodicity of a face-centered cubic lattice. Next, we demonstrated the biocompatibility of the structures by observing the viability of cells cultured on them. We finally demonstrated that neuronlike PC12 cells grow on and within such stacked microscaffolds and outgrow neurites through cage boundaries as a first step towards controlled 3D cell networks.

The novel technique can be used to stack different types of cells layer by layer and thus to take advantage of the interaction between different cell types. We envision applications in cell biology, lab-on-a-chip systems, and artificial heterogeneous tissue development, among others. The technique may also be of interest for applications of organs-on-a-chip designers where microscaffolds for controlled cell growth can possibly be integrated with more complex microfluidic systems.

\section{Acknowledgments}

This research was funded by the EU 7th Framework Programme under the EUJO-LIMMS project, grant agreement $n^{\circ} 295089$. Z. Fekete is grateful for the support of the Alexander von Humboldt Foundation.

\section{References}

[1] Y. Yi, J. Park, J. Lim, C. J. Lee, S. H. Lee, Trends Biotechnol. 2015, 33, 762. 
[2] Y. J. Choi, S. Chae, J. H. Kim, K. F. Barald, J. Y. Park, S.-H. Lee, Sci. Rep. 2013, 3, 1921.

[3] H. T. Tran, C. H. Y. Chung, M. Iba, B. Zhang, J. Q. Trojanowski, K. C. Luk, V. M. Y. Lee, Cell Rep. 2014, 7, 2054.

[4] M. S. Feiler, B. Strobel, A. Freischmidt, A. M. Helferich, J. Kappel, B. M. Brewer, D. Li, D. R. Thal, P. Walther, A. C. Ludolph, K. M. Danzer, J. H. Weishaupt, J. Cell Biol. 2015, 211, 897.

[5] M. Hasan, Y. Berdichevsky, Micromachines, 2016, 7, 157.

[6] M. J. Aebersold, H. Dermutz, C. Forró, S. Weydert, G. Thompson-Steckel, J. Vörös, L. Demkó, Trends Anal. Chem., 2016, 78, 60.

[7] T. Honegger, M. I. Thielen, S. Feizi, N. E. Sanjana, J. Voldman, Sci. Rep., 2016, 6, 28384.

[8] C. Tomba, C. Villard, Micro. Engn., 2015, 132, 176-191.

[9] S. Yoshida, T. Teshima, K. Kuribayashi-Shigetomi, S. Takeuchi, Adv. Healthcare Mater., 2016, $5,415-420$.

[10] F. Larramendy, M. C. Blatche, L. Mazenq, A. Laborde, P. Temple-Boyer, O. Paul, J. Micromech. Microeng., 2015, 25, 1-9.

[11] D. Huh, G. A. Hamilton, D. E. Ingber, Trends Cell Biol., 2011, Vol. 21, 12, 745-754.

[12] H. Aubin, J. W. Nichol, C. B. Hutson, H. Bae, A. L. Sieminski, D. M. Cropek, P. Akhyari, A. Khademhosseini, Biomaterials, 2010, 31, 6941-6951.

[13] S. I. Fraley, Y. Feng, R. Krishnamurthy, D.-H. Kim, A. Celedon, G. D. Longmore, D. Wirtz, Nat. Cell Biol., 2010, 12, 598 - 604.

[14] A. M. Greiner, B. Richter, M. Bastmeyer, Micromol. Biosci., 2012, 12, 1301-1314.

[15] A. Kunze, M. Giugliano, A. Valero, P. Renaud, Biomaterials, 2011, 32, 2088-2098.

[16] S.-A. Lee, D. Y. No, E. Kang, J. Ju, D.-S. Kim, S.-H. Lee, Lab Chip, 2013, 13, 3529.

[17] D. W. Lee, Y.-S. Choi, Y. J. Seo, M.-Y. Lee, S. Y. Jeon, B. Ku, S. Kim, S. H. Yi, D.-H. Nam, Anal. Chem., 2013, 86, 335.

[18] V.N. Goral, S.H. Au, R.A. Faris, P.K. Yuen, Lab on a Chip, 2015, 15, 1032-1037.

[19] E. O. Mosaad, K. F. Chambers, K. Futrega, J.A. Clements, M.R. Doran, M. R. Scientific reports, 2018, 8, 253. 
[20] X.F. Tian, B.C. Heng, Z. Ge, K. Lu, A.J. Rufaihah, V.T. Fan, J.F. Yeo, T. Scand. J. Clin. Lab. Invest., 2008, 68, 58-67

[21] Y. Imamura, T. Mukohara, Y. Shimono, Y. Funakoshi, N. Chayahara, M. Toyoda, N. Kiyota, S. Takao, S. Kono, T. Nakatsura, H. Minami, Oncol. Rep., 2018, 33, 1837-1843

[22] N. Takahashi, T., Sasaki, W. Matsumoto, N. Matsuki, Y. Ikegaya, Proc. Natl. Acad. Sci. USA 2010, 107, 10244-10249.

[23] S. Bosi et al., Sci. Rep., 2015, 5, 9562.

[24] F.P. Severino, J. Ban, Q. Song, M. Tang, G. Bianconi, G. Cheng, V. Torre, Sci. Rep., 2016, 6, 29640.

[25] D. Kim, S-M. Kim, S. Lee, M-H. Yoon, Sci. Rep., 2017, 7, 7716.

[26] S. Li, F.P.U. Severino, J. Ban, L. Wang, G. Pinato, V. Torre, Y. Chen, Biomed. Mat., 2018, 13, 034105.

[27] D. Huh, G.A. Hamilton, D.E. Ingber, Trends Cell Biol., 2011, 21, 745-54.

[28] S. Breslin, L. O’Driscoll, Drug Discovery Today, 2013, 18, 240-249.

[29] M. Kato-Negishi, Y. Morimoto, H. Onoe, S. Takeuchi, Adv. Healthcare Mater., 2013, 2, 1564.

[30] J. Park, B. K. Lee, G. S. Jeong, J. K. Hyun, C. J. Lee, S.-H. Lee, Lab Chip, 2015, 15, 141-150.

[31] T. Xu, P. Molnar, C. Gregory, M. Das, T. Boland, J. J. Hickman, Biomaterials, 2009, 30, $4377-4383$.

[32] R. Lozano, L. Stevens, B. C. Thompson, K. J. Gilmore, R. Gorkin III, E. M. Stewart, M. in het Panhuis, M. Romero-Ortega, G. G. Wallace, Biomaterials, 2015, 67, 264-273.

[33] M. D. Tang-Schomer, J. D. White, L. W. Tien, L. I. Schmitt, T. M. Valentin, D. J. Graziano, A. M. Hopkins, F. G. Omenetto, P. G. Haydon, D. L. Kaplan, $P N A S, 1$ 2014, 11, 13811-13816.

[34] N. Li, Q. Zhang, S. Gao, Q. Song, R. Huang, L. Wang, L. Liu, J. Dai, M. Tang, G. Cheng, Sci. Rep., 2013, 3, 1604.

[35] S. Bosi, R. Rauti, J. Laishram, A. Turco, D. Lonardoni, T. Nieus, M. Prato, D. Scaini, L. Ballerini, Sci. Rep., 2015, 5, 9562.

[36] B. Spagnolo, V. Brunetti, G. Leménager, E. De Luca, L. Sileo, T. Pellegrino, P. P. Pompa, M. De Vittorio \& Ferruccio Pisanello, Sci. Rep., 2015, 5, 10531. 
[37] Y. J. Choi, J. Park, S.-H. Lee, Biomaterials, 2013, 34, 2938-2946.

[38] F. Larramendy, S. Yoshida, L. Jalabert, S. Takeuchi, O. Paul, J. Micromech. Microeng., 2016, $26,095017$.

[39] M. Frega, M. Tedesco, P. Massobrio, M. Pesce, S. Martinoia, Sci. Rep., 2014, 4, 5489.

[40] J. M. Singelyn, J. A. DeQuach, S. B. Seif-Naraghi, R. B. Littlefield, P. J. Schup-Magoffin, K. L. Christman, Biomaterials, 2009, 30, 5409-5416.

[41] M. R. Gullo, S. Takeuchi, O. Paul, Adv. Healthcare Mater., 2017, 6, 1601053.

[42] X. X. Shao, D. W. Hutmacher, S. T. Ho, J. C. H. Goh, E. H. Lee, Biomaterials, 2006, 27 (7), 1071-1080.

[43] B. B. Mandal, S.C. Kundu, Biomaterials, 2009, 30, 2956-2965.

[44] S. Wu, J. Serbin, M. Gu, J. Photoch. Photobio. A, 2006, 181, 1-11.

[45] K.-S. Lee, D.-Y. Yang, S. H. Park, R. H. Kim, Polym. Adv. Technol., 2006, 17, 72-82.

[46] M. T. Raimondi, S. M. Eaton, M. Laganà, V. Aprile, M. M. Nava, G. Cerullo, R. Osellame, Acta Biomater., 2013, 9, 4579-4584.

[47] M. H. Olsen, G. M. Hjortø, M. Hansen, Ö. Met, I. M. Svane, N. B. Larsen, Lab Chip, 2013, 13, 4800-4809.

[48] M. Mihailescu, I. A. Paun, M. Zamfirescu, C. R. Luculescu, A. M. Acasandrei, M. Dinescu, J. Mater. Sci., 2016, 51, 4262-4273.

[49] F. Larramendy, S. Yoshida, Z. Fekete, D. Serien, S. Takeuchi, O. Paul, Transducers 2015, Anchorage, Alaska, USA.

[50] N. W. Ashcroft, N. D. Mermin, Solid State Physics $1^{\text {st }}$ Edition, Saunders College Division, 1976, ISBN13: 978-0030839931 ISBN10: 0030839939.

[51] V. N. Vernekar, D. K. Cullen, N. Fogleman, Y. Choi, A. J. Garcia, M. G. Allen, G. J. Brewer, M. C. LaPlaca, J. Biomed. Mater. Res., 2009, 89A, 138-151.

[52] F. Larramendy, A. Bendali, M. C. Blatché, F. Mathieu, S. Picaud, P. Temple-Boyer, L. Nicu, Sensor. Actuat. B-Chem., 2014, 203, 375-381. 
[53] J. J. Bozzola, L. D. Russel, Electron Microscopy Principles and Techniques for Biologists, Jones and Bartlett Publishers, Boston 1999.

[54] S. Yoshida, K. Sato, S. Takeuchi, The 27th IEEE International Conference on Micro Electro Mechanical Systems, 2014.

[55] N. A. Bakhtina, U. Loeffelmann, N. MacKinnon, J. G. Korvink, Adv. Funct. Mater., 2015, $25,1683-1693$.

[56] B. Li, M. Liu, Q. Chen, J. Micro/Nanolith. MEMS MOEMS, 2005, 4 (4), 043008-6 .

[57] E. H. Conradie, D. F. Moore, J. Micromech. Microeng., 2002, 12, 368-374.

[58] J. Hammacher, A. Fuelle, J. Flaemig, J. Saupe, B. Loechel, J. Grimm, Microsyst. Technol., 2008, 14, 1515-1523.

[59] K.Wouters, R. Puers, J. Micromech. Microeng., 2010, 20, 095013.

[60] C. Gruber, A. Hirzer, V. Schmidt, A. Trügler, U. Hohenester, H. Ditlbacher, A. Hohenau, J. R. Krenn, Appl. Phys. Lett., 2015, 106, 081101.

[61] M. Al-Abaddi, L. Sasso, M. Dimaki, W. E. Svendsen, Micro. Engn., 2012, 98, 378-381. 


\section{Figure Caption}

Figure 1. Concept of micro-cages for 3D controlled celullar network (a) inspired by the geometry of the truncated octahedron (b) cut into two identical structures. (c) Both structure was created to be robust by thickening their edges; connection holes and pins were added to create type $A$ and type $B$ structures. (d) Initial structures are reproduced periodically in 2D and cell barriers were added to obtain $2 \times 2$ unit structures. (e,f) Larger structures used in the experiments are built from these unit structures, with cuboid blocks added on their sides for manipulation and alignment.

Figure 2. Fabrication process of micro-cages for 3D controlled cellular network: (a) glass substrate with IP-L 780 photoresist drop; (b) structures defined by two-photon lithography; (c) the culture chamber defined by a silicone rubber frame; all surfaces were coated with collagen; (d) growth medium solution is added before (e) seeding PC12 cells on the structure; (f) finally, the structures are stacked, allowing the $3 D$ growth of a cell network.

Figure 3. SE micrographs of microstructures with (a) 16 and (b) 32 containers. (c) Two-layer stack enabled by (d) lateral alignment blocks. (e) Close-up of cell cages.

Figure 4. SE micrographs of (a) a structure developed in IPA solution for $20 \mathrm{~min}$, showing significant mechanical stress and a structure developed in PGMEA developer for 20 min and rinsed with IPA, without apparent stress.

Figure 5. (a-d) Confocal micrographs of structure (blue fluorescence) with cultured cells (green fluorescence) after 2 days of culture on structures with different container dimensions. Container diameters: (a) $30 \mu \mathrm{m}$, (b) $40 \mu \mathrm{m}$, (c) $50 \mu \mathrm{m}$, and (d) $60 \mu \mathrm{m}$. (e) Bar graph of the number of cells per container, for (Green) no cell, (Blue) one cell, (Orange) two cells, and (Red) equal or superior to three cells, for a maximum of 32 containers, for container diameters of $30 \mu \mathrm{m}, 40 \mu \mathrm{m}, 50 \mu \mathrm{m}$, and $60 \mu \mathrm{m}$. Error bars indicate the maximum and minimum for each configuration.

Figure 6. Optical micrographs (gray) of cells on glass substrate and on structure with collagen coating after 2, 7, and 16 days. Results of live (green) and dead (red) tests after 16 days of cell culture show a large numbers of live cells, especially on the structure. 
Figure 7. Confocal optical micrographs of (a) live cells (bright green), (b) dead cells (red) on (c) three stacked structures revealed by fluorescence and (d) the combination of the three fluorescence images showing the live and dead cells through the stacked structures.

Figure 8. SE micrographs of (a) a tri-layer stack of structures with cells, (b) two four-layer stacks with cells (c) neurites bridging layers and thereby creating $3 D$ neuronal networks (denoted by red arrows).

\section{Author Contributions Statement}

F.L., S.Y., Z.F., O.P., S.T. wrote the main manuscript and prepared the figures. Z.F., D.M., and F.L. worked on the optimization of two-photon polymerization and microfabrication. S.Y., and F.L. performed scaffold assembly, SEM analysis and in vitro experiments. All authors reviewed the manuscript.

\section{Competing financial and non-financial interests}

The authors declare no competing financial and non-financial interests. 

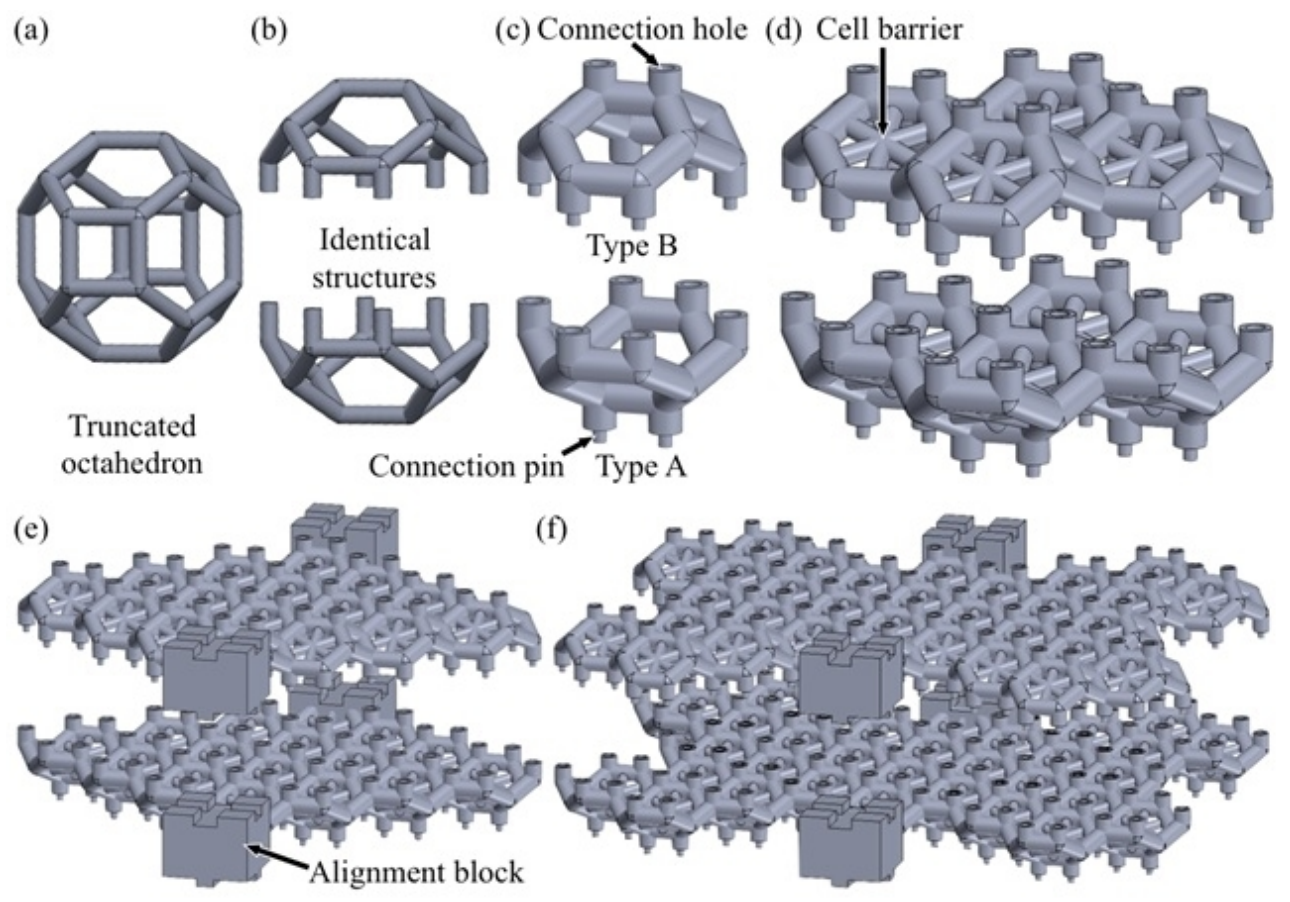

Concept of micro-cages for 3D controlled celullar network (a) inspired by the geometry of the truncated octahedron (b) cut into two identical structures. (c) Both structure was created to be robust by thickening their edges; connection holes and pins were added to create type A and type B structures. (d) Initial structures are reproduced periodically in $2 \mathrm{D}$ and cell barriers were added to obtain $2 \times 2$ unit structures. (e,f) Larger structures used in the experiments are built from these unit structures, with cuboid blocks added on their sides for manipulation and alignment.

$173 \times 120 \mathrm{~mm}(96 \times 96 \mathrm{DPI})$ 
(a)

IP-L 780 photoresist drop

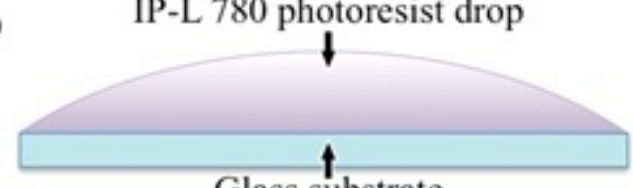

Glass substrate

(b) Type A and type B structural layer

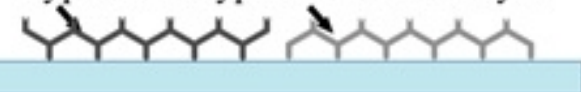

(c) Silicone rubber Collagen solution

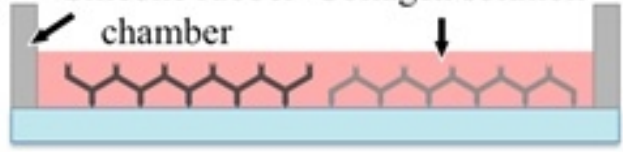

(d)

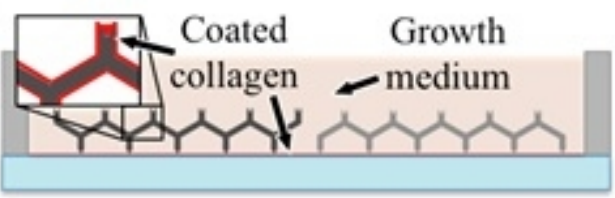

(e)
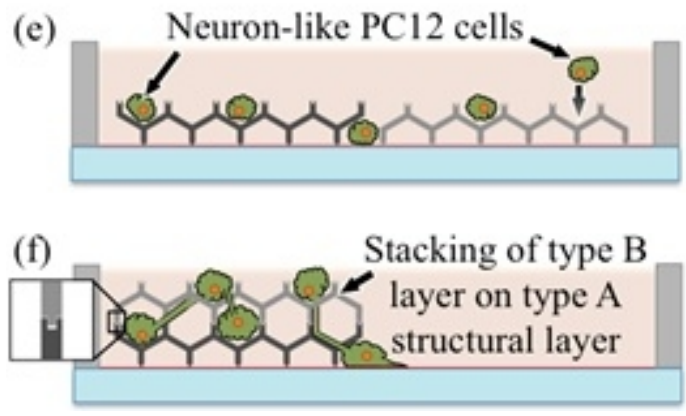

Fabrication process of micro-cages for 3D controlled cellular network: (a) glass substrate with IP-L 780 photoresist drop; (b) structures defined by two-photon lithography; (c) the culture chamber defined by a silicone rubber frame; all surfaces were coated with collagen; (d) growth medium solution is added before (e) seeding PC12 cells on the structure; (f) finally, the structures are stacked, allowing the 3D growth of a cell network. 

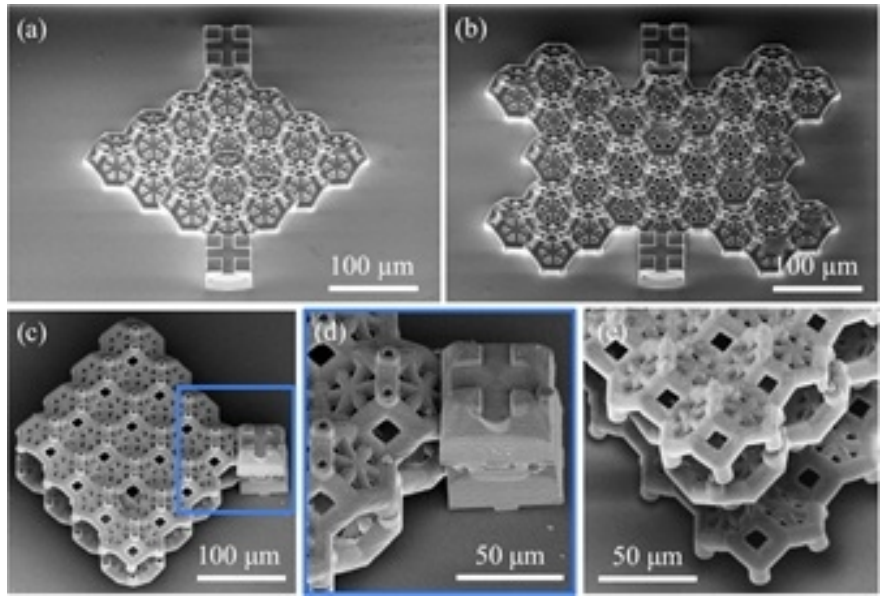

. SE micrographs of microstructures with (a) 16 and (b) 32 containers. (c) Two-layer stack enabled by (d) lateral alignment blocks. (e) Close-up of cell cages.

$88 \times 60 \mathrm{~mm}(96 \times 96 \mathrm{DPI})$ 


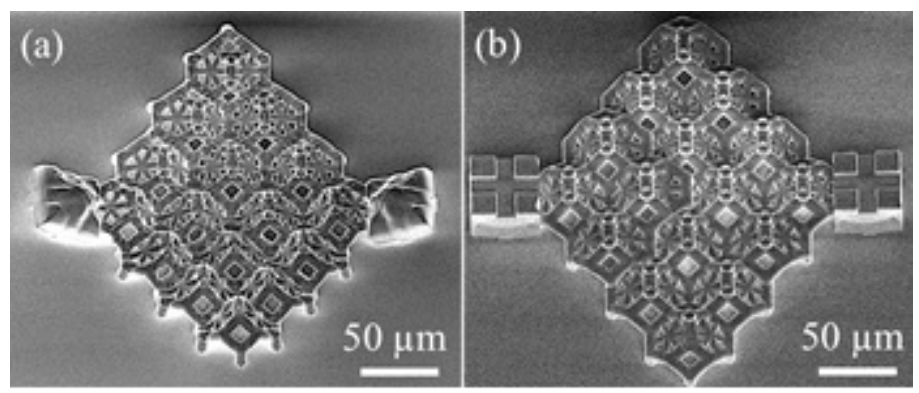

SE micrographs of (a) a structure developed in IPA solution for 20 min, showing significant mechanical stress and a structure developed in PGMEA developer for $20 \mathrm{~min}$ and rinsed with IPA, without apparent stress.

$91 \times 38 \mathrm{~mm}(96 \times 96 \mathrm{DPI})$ 

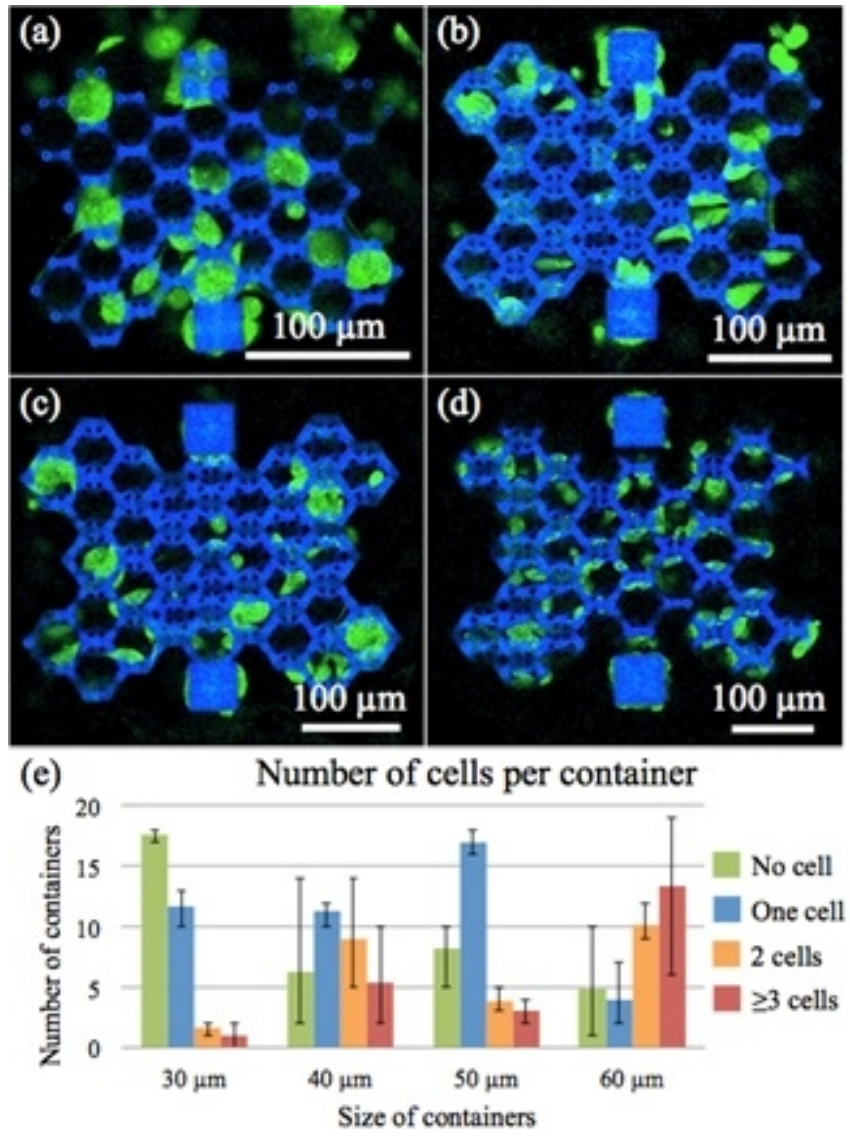

(a-d) Confocal micrographs of structure (blue fluorescence) with cultured cells (green fluorescence) after 2 days of culture on structures with different container dimensions. Container diameters: (a) $30 \mu \mathrm{m}$, (b) 40 $\mu \mathrm{m}$, (c) $50 \mu \mathrm{m}$, and (d) $60 \mu \mathrm{m}$. (e) Bar graph of the number of cells per container, for (Green) no cell, (Blue) one cell, (Orange) two cells, and (Red) equal or superior to three cells, for a maximum of 32 containers, for container diameters of $30 \mu \mathrm{m}, 40 \mu \mathrm{m}, 50 \mu \mathrm{m}$, and $60 \mu \mathrm{m}$. Error bars indicate the maximum and minimum for each configuration.

$86 \times 116 \mathrm{~mm}(96 \times 96 \mathrm{DPI})$ 


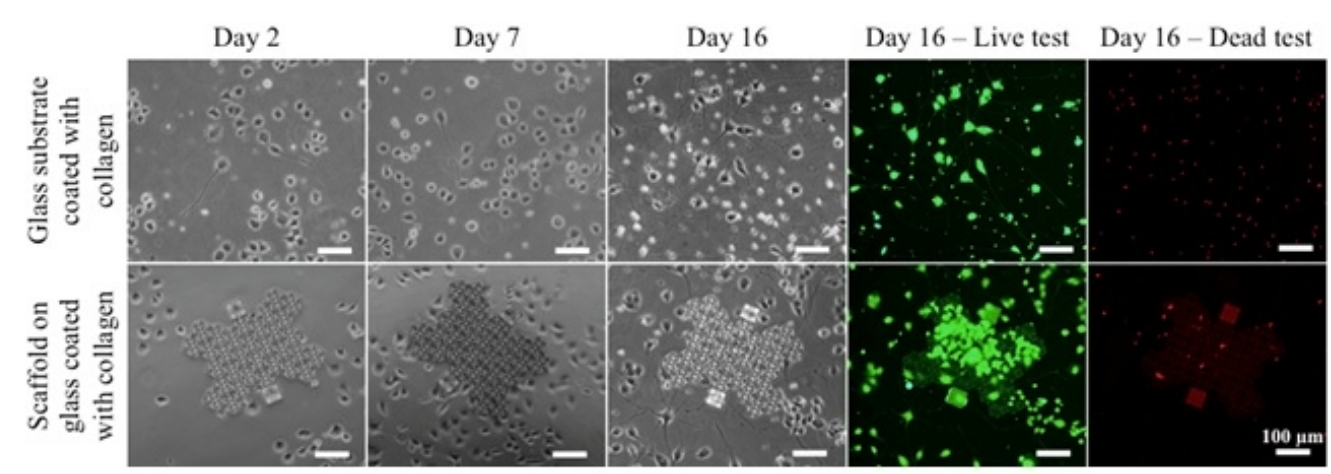

Optical micrographs (gray) of cells on glass substrate and on structure with collagen coating after 2, 7, and 16 days. Results of live (green) and dead (red) tests after 16 days of cell culture show a large numbers of live cells, especially on the structure.

$169 \times 59 \mathrm{~mm}(96 \times 96 \mathrm{DPI})$ 

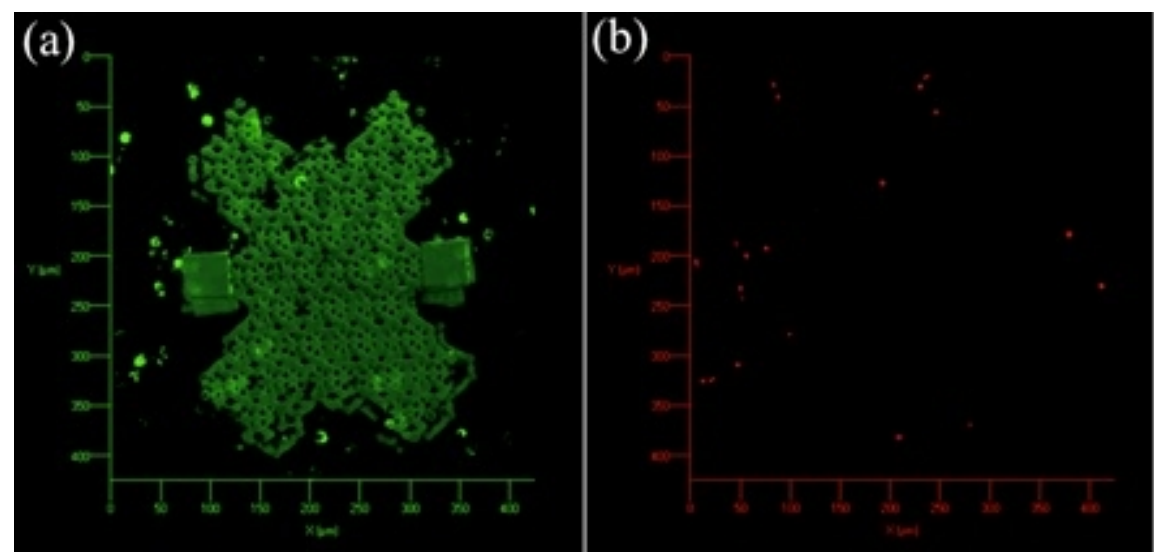

(c)

(d)

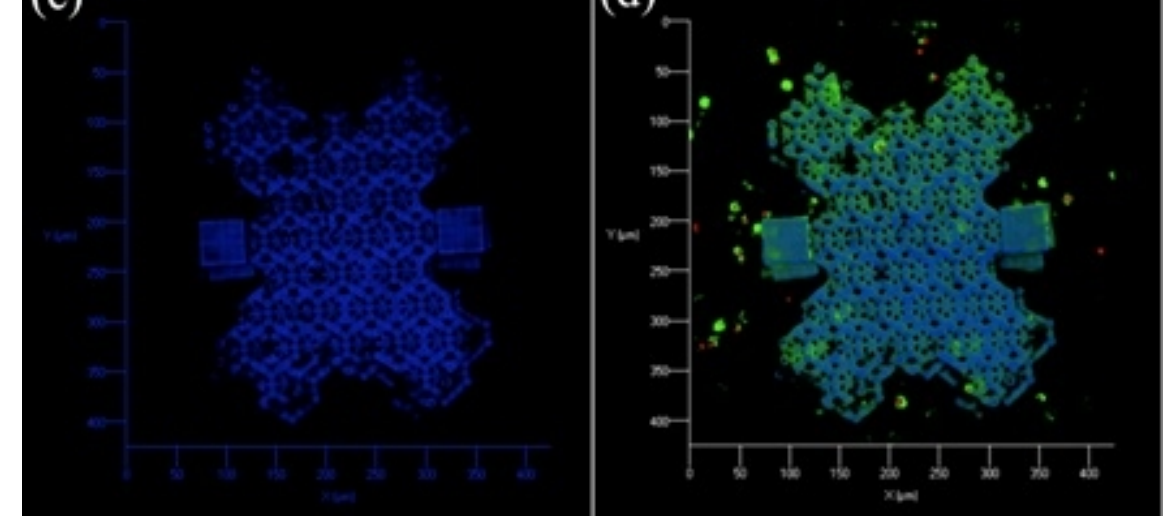

Confocal optical micrographs of (a) live cells (bright green), (b) dead cells (red) on (c) three stacked structures revealed by fluorescence and (d) the combination of the three fluorescence images showing the live and dead cells through the stacked structures.

$115 \times 111 \mathrm{~mm}(96 \times 96 \mathrm{DPI})$ 

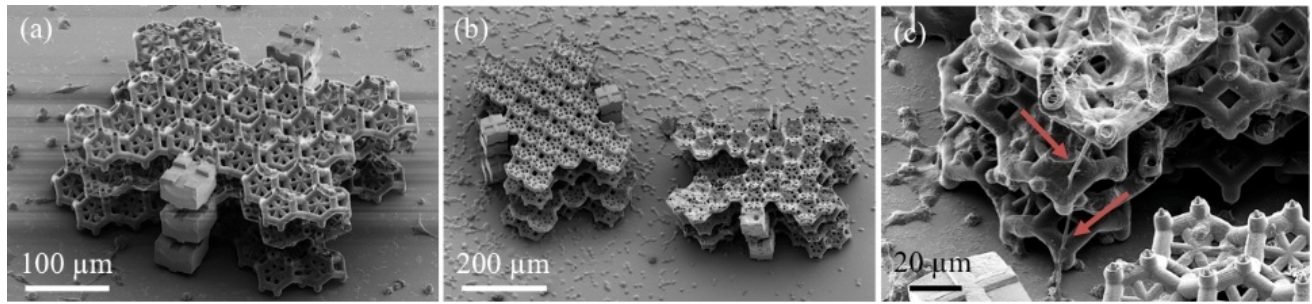

SE micrographs of (a) a tri-layer stack of structures with cells, (b) two four-layer stacks with cells (c) neurites bridging layers and thereby creating 3D neuronal networks (denoted by red arrows).

$254 \times 58 \mathrm{~mm}(96 \times 96 \mathrm{DPI})$ 